\title{
Implication of plasma intermedin levels in patients who underwent first-time diagnostic coronary angiography: a single centre, cross-sectional study
}

Aylin Hatice Yamac, Ahmet Bacaksiz, Ziya Ismailoglu, Sitki Kucukbuzcu, Emrah Sevgili, Emin Asoglu, Muharrem Nasifov, Parviz Jafarov, Ercan Erdogan and Omer Goktekin

\begin{abstract}
Background: Intermedin (IMD) is involved in the prevention of atherosclerotic plaque progression, possessing cardioprotective effects from hypertrophy, fibrosis and ischemia-reperfusion injury. Elevated plasma IMD levels have been demonstrated in patients with acute coronary syndromes. No human study has examined the role of IMD in stable patients who underwent diagnostic coronary angiography with suspicion of coronary artery disease (CAD). Thus we investigated the role of IMD as a biomarker to discriminate patients with CAD and predict those with severe disease who require early and intensive therapeutic intervention before presenting with acute coronary syndrome.
\end{abstract}

Methods: Eligible two hundred and thirty-eight consecutive patients (123 males, mean age $58.4 \pm 10.0$ years) who underwent first-time diagnostic coronary angiography were included in this study. Plasma concentrations of IMD were measured from arterial blood samples by the enzyme-linked immunosorbent assay. Patients were divided into three groups according to the presence and degree of CAD, consisting of 48 patients with normal coronary anatomy (Group 1), 111 patients with $<50 \%$ coronary stenosis (Group 2), and 79 patients with $\geq 50 \%$ stenosis in at least one of the major coronary arteries (group 3). The severity and extent of CAD was evaluated by calculations of the vessel, Gensini, and SYNTAX scores.

Results: Circulating plasma IMD levels in patients with CAD were significantly higher than those in patients without CAD (157.7 $\pm 9.6,134.8 \pm 11.9$, and $117.6 \pm 7.9 \mathrm{pg} / \mathrm{mL}$ in groups 3,2 and 1 respectively; $p<0.001)$. Besides, plasma IMD levels were correlated with Gensini and SYNTAX scores $\left(r_{s}=0.742\right.$, and $r_{s}=0.296$, respectively; $\left.p<0.05\right)$. The presence of $\geq 50 \%$ coronary artery stenosis could be predicted if a cut-off value of $147.7 \mathrm{pg} / \mathrm{mL}$ for plasma IMD was used with $88.6 \%$ sensitivity and $88.7 \%$ specificity. Moreover, a plasma IMD level of $<126.6 \mathrm{pg} / \mathrm{mL}$ could discriminate a patient with normal coronary arteries from patients with angiographically proven CAD with a sensitivity and specificity of $84.7 \%$, and $83.3 \%$ respectively.

Conclusions: We demonstrated that IMD might be used as a biomarker to predict CAD and its severity in patients who underwent first time diagnostic coronary angiography.

Keywords: Intermedin, Coronary artery disease, Diagnostic coronary angiography

\footnotetext{
* Correspondence: haticeyamac80@yahoo.de

Faculty of Medicine, Department of Cardiology, BezmiÂlem Foundation University, Adnan Menderes Avenue, Vatan Street, 34093 Fatih, Istanbul, Turkey
} 


\section{Background}

Cardiovascular disease is the leading cause of morbidity and mortality worldwide [1]. Although atherosclerosis is the underlying pathology in general, there are many mechanisms proposed to explain the development of the disease from plaque formation to thrombus related occlusion of the artery such as, inflammation, oxidative stress, and endothelial dysfunction $[2,3]$. Identification and quantification of atherosclerotic involvement is of high importance to determine patients at high risk for future cardiovascular events. Nowadays, there is considerable evidence supporting the clinical utility of several biomarkers, which are involved in the development of atherogenesis, to determine the prospective cardiovascular risk [4].

Intermedin (IMD) is a novel member of the calcitonin gene-related peptide (CGRP) family, which serves as an endocrine integrator of homeostasis in the cardiovascular and renal systems [5]. It exerts directly positive inotropic and chronotropic effects on the myocardium, besides IMD reduces the cardiac afterload via systemic and pulmonary vasodilatation, induces coronary vasodilatation, thereby enhancing myocardial perfusion [6]. In addition, it has been also reported to be cardioprotective from remodelling by inhibiting cardiac fibroblast activation induced by angiotensin II, anti-hypertrophic in conditions that promote cardiac myocyte hypertrophy, and protecting against ischemia-reperfusion injury by inhibiting formation of reactive oxygen species $[5,7,8]$. Furthermore, Zhang et al. demonstrated that exogenous administration of IMD could prevent the progression of atherosclerotic plaque formation in ApoE null mice [9]. Two recent human studies, which investigated the role of IMD in acute coronary syndromes, demonstrated that plasma levels of IMD were markedly elevated in patients with acute myocardial infarction and moreover revealed that it could be used as a marker to reflect the severity of coronary stenosis in this patient collective $[10,11]$.

To the best of our knowledge, there is not any data about plasma IMD concentrations and its diagnostic value in stable patients with signs and/or symptoms suggesting coronary artery disease (CAD). The aim of this study is to investigate the clinical relevance of plasma IMD levels to predict CAD in patients who underwent first-time diagnostic coronary angiography and its association with the severity and extent of the disease.

\section{Methods}

\section{Study population}

Patients enrolled in this study were patients who underwent diagnostic conventional coronary angiography for suspected CAD at our institution between February 1, 2013 and November 1, 2013. Exclusion criteria included histories of recent myocardial infarction (MI), unstable angina pectoris (UAP), heart failure (systolic and/or diastolic heart failure was excluded with the use of transthoracic echocardiography, left ventricular ejection fraction $<40 \%$ was accepted as systolic heart failure), moderate to severe heart valve disease, malignancies, major trauma or surgery in the previous six months, renal insufficiency, acute or chronic infectious disease, chronic obstructive pulmonary disease, any kind of immune-mediated disease, since all these conditions may affect plasma IMD levels. Patients with coronary anomalies and/or slow flow phenomenon were also excluded. According to the inclusion criteria two hundred and thirty-eight eligible patients were included into the study. The local ethics committee (BezmiÂlem Foundation University Clinical Studies Ethics Committee) approved the study protocol, and all patients provided an informed consent. The study was conducted in accordance with the ethical principles described by the Declaration of Helsinki.

\section{Coronary angiography}

Coronary angiography was performed by a monoplane cine-angiography system. Standard selective coronary angiography with at least four views of the left coronary system and two views of the right coronary artery had been performed using the Judkins technique. All angiograms were recorded at a 25 frames/second rate by using a $35 \mathrm{~mm}$ cinefilm and iopromide (Ultravist 370, Schering AG, Berlin, Germany) was used as opaque material. Two blinded experienced interventional cardiologists from our institute examined the angiograms. When there was a disagreement, the difference was adjusted by a third investigator.

Modified Gensini scoring system and the vessel score were used to determine the severity and extent of CAD $[12,13]$. To calculate the vessel score, one point was assigned to each coronary artery when there was $50 \%$ or more stenosis in the three main coronary arteries and their major side branches [13]. One additional point was assigned when there was $50 \%$ or more stenosis in the left main coronary artery (LMCA), consequently with a total score of maximum 4. On the other hand, modified Gensini score was obtained by giving consideration to the severity of lesions at certain segments of the LMCA, left anterior descending (LAD), circumflex $(\mathrm{Cx})$ and right coronary (RCA) arteries [12]. Two points were assigned for a lesion with an obstruction level between $0-24 \%, 4$ points for a lesion of $25-49 \%, 8$ points for a lesion of 50 $74 \%, 16$ points for a lesion of $75-89 \%, 32$ points for a lesion of $90-99 \%$ and 64 points for a lesion of $100 \%$ obstruction. If the RCA was dominant, the lesion score for LMCA was multiplied by a constant multiplier of 5 ; the lesion scores for proximal LAD and $\mathrm{Cx}$ were multiplied by 2.5 ; the score for medial LAD lesion was multiplied by 1.5 ; the scores for RCA, distal LAD and distal $\mathrm{Cx}$ lesions were multiplied by 1 ; the scores for optus 
marginalis (OM1) and diagonal (D1) side branches were multiplied by 1 and the lesion score for other side branches was multiplied by 0.5 . Total Gensini score was obtained by adding the resultant figures. If the left system was dominant, a constant multiplier of 0.5 multiplied the scores of proximal, medial and distal segment lesions of the RCA. Ultimately, total Gensini score was obtained numerically, indicating the severity of CAD. In addition, the Synergy between PCI with Taxus and Cardiac Surgery (SYNTAX) score was applied to all coronary lesions with a diameter stenosis greater than $50 \%$ in vessels with a dimater larger than $1.5 \mathrm{~mm}$ as described before [14]. Inter- and intra-observer variability for repeated evaluations of angiograms of 20 randomly selected patients was low (for Gensini score: interobserver variability $4.1 \pm 1.9 \%$; intra-observer variability $4.4 \pm 2.0 \%)$.

\section{Laboratory tests}

Peripheral blood samples for complete blood count and serum creatinine, fasting glucose and lipid profiles were taken from an antecubital vein in the morning before the coronary angiography. All patients had normal serum creatinine levels and normal white blood cell counts. Total cholesterol, triglycerides, and high-density lipoprotein cholesterol were measured using enzymatic methods after overnight fasting. Low density lipoprotein (LDL) cholesterol concentrations were calculated using the Friedewald formula [15].

Arterial blood samples for IMD measurements were collected in EDTA anticoagulant tubes from the arterial sheath before coronary angiography. The samples were centrifuged for 10 minutes at $4{ }^{\circ} \mathrm{C}$ and $3000 \mathrm{~g}$ within $30 \mathrm{mi}-$ nutes after collection for separation of plasma. All plasma samples were stored frozen at $-40^{\circ} \mathrm{C}$ before use. Plasma concentrations of IMD were measured by the enzymelinked immunosorbent assay (ELISA) kit, manufactured by MyBioSource, Inc. (San Diego, USA). The intra- and inter-assay coefficient of variation was less than $10 \%$ and $5 \%$ respectively. All procedures followed strictly the manufacturer's instructions.

\section{Statistical analysis}

Data were analyzed by using the SPSS software version 13.0 (SPSS, Chicago, IL). The distribution of the variables was analyzed with the Kolmogorov-Smirnow test. Continuous, normally distributed variables were presented as mean \pm standart deviation (SD), and non-normally distributed variables as median (interquartile range). Categorical and ordinal data were presented as frequencies and/or percentages. Differences between groups were tested using Student's t test or Mann-Whitney U test. Categorical variables were compared by the $\chi^{2}$-test. The Spearman's correlation coefficient $\left(r_{s}\right)$ was computed to assess variable relations. A regression analysis was performed to detect the predictors of presence of angiographically confirmed CAD. The univariated regression model was used separately for each following covariates: age, gender, smoking status, BMI, hypertension, diabetes, dyslipidemia, and serum IMD level. The covariates, which were significantly associated with the presence of CAD in the univariated model, were included into the multivariate logistic regression analysis. A two-sided $p$ value $<0.05$ was considered statistically significant.

\section{Results}

Patients' baseline characteristics were listed in Table 1 . Patients were divided into three groups according to the presence and degree of luminal stenosis on coronary angiography: 48 patients with normal coronary anatomy (group 1), 111 patients with $<50 \%$ coronary stenosis (group 2), and 79 patients with $\geq 50 \%$ stenosis in at least one of the major coronary arteries (group 3). Patients with CAD (groups 2 and 3 ) were mostly males and they were significantly older than patients in group $1 \quad(p<0.05)$. There was no significant difference in terms of BMI and waist circumference. Besides, smokers were more common in group 2, but the difference did not reach any statistical significance. However, traditional cardiovascular risk factors, such as hypertension, diabetes, and hyperlipidemia were not different in patients with and without CAD. Laboratory measures, including serum glucose, creatinine and WBC, were similar in both groups. Patients in group 3 had higher LDL-cholesterol levels compared with patients in group $1(149.1 \pm 37.6$ versus $130.1 \pm 28.9 \mathrm{mg} / \mathrm{dL}$, respectively; $p<0.05$ ).

Male patients had higher plasma IMD levels compared to females $(142.3 \pm 16.8$ versus $135.3 \pm 18.7 \mathrm{pg} / \mathrm{mL}$, respectively; $p<0.01$ ). In addition, the plasma IMD concentration was elevated in current smokers $(143.4 \pm 16.8$ versus $137.3 \pm 18.3 \mathrm{pg} / \mathrm{mL}$, respectively; $p=0.02$ ). Presence of other cardiovascular risk factors such as hypertension, diabetes mellitus, and hyperlipidemia did not affect plasma IMD levels (Table 2). A positive correlation was observed between plasma IMD levels and age $\left(\mathrm{r}_{\mathrm{s}}=0.255, p<0.01\right)$.

The plasma IMD concentration in patients with CAD was significantly higher than in patients without CAD $(157.7 \pm 9.6,134.8 \pm 11.9$, and $117.6 \pm 7.9 \mathrm{pg} / \mathrm{mL}$ in Groups 3, 2, and 1, respectively; $p<0.01$ ) (Table 3, Figure 1). In addition, plasma IMD levels were correlated with the vessel, Gensini, and SYNYAX scores $\left(r_{s}=0.710\right.$, $\mathrm{rs}=0.742$, and $\mathrm{r}_{\mathrm{s}}=0.296$, respectively; $p<0.01$ ) (Figure 2a, $\mathrm{b}$, and $\mathrm{c}$ ).

ROC curve was generated for sensitivity and specificity with the respective areas under the curve (AUC) for the plasma IMD concentration. The diagnostic value for plasma IMD levels in discriminating patients with $\geq 50 \%$ coronary stenosis in at least one of the coronary arteries 
Table 1 Patient demographics, clinical and laboratory characteristics

\begin{tabular}{|c|c|c|c|}
\hline & $\begin{array}{l}\text { Patients without CAD } \\
\text { (Group 1) }(n=48)\end{array}$ & $\begin{array}{l}\text { Patients with }<50 \% \text { coronary stenosis } \\
\text { (Group 2) }(n=111)\end{array}$ & $\begin{array}{l}\text { Patients with } \geq 50 \% \text { coronary stenosis } \\
\text { (Group 3) }(n=79)\end{array}$ \\
\hline Age (years) & $52.5 \pm 8.8$ & $58.0 \pm 9.9^{*}$ & $62.6 \pm 9.1^{* \#}$ \\
\hline Male gender (\%) & $14(29.2 \%)$ & $54(48.6 \%)^{*}$ & $55(69.6 \%)^{* \#}$ \\
\hline $\mathrm{BMI}\left(\mathrm{kg} / \mathrm{m}^{2}\right)$ & $30.6 \pm 4.6$ & $29.0 \pm 4.9$ & $28.7 \pm 5.1$ \\
\hline Waist circumference $(\mathrm{cm})$ & $100.9 \pm 10.2$ & $101.4 \pm 9.2$ & $102.1 \pm 8.2$ \\
\hline Current smoker & $10(20.8 \%)$ & $28(25.2 \%)$ & $26(32.9 \%)$ \\
\hline Hypertension (\%) & $23(47.9 \%)$ & $61(54.9 \%)$ & $34(43.0 \%)$ \\
\hline Diabetes mellitus (\%) & $12(25.0 \%)$ & $35(31.5 \%)$ & $24(30.4 \%)$ \\
\hline Dyslipidemia (\%) & $10(20.8 \%)$ & $28(25.2 \%)$ & $16(20.2 \%)$ \\
\hline $\mathrm{SBP}(\mathrm{mmHg})$ & $135.8 \pm 22.6$ & $138.0 \pm 22.8$ & $137.5 \pm 20.9$ \\
\hline $\mathrm{DBP}(\mathrm{mmHg})$ & $78.2 \pm 8.3$ & $79.2 \pm 10.5$ & $79.5 \pm 9.3$ \\
\hline Glucose (mg/dL) & $118.2 \pm 57.5$ & $114.9 \pm 31.1$ & $117.9 \pm 44.4$ \\
\hline Creatinine (mg/dL) & $0.7 \pm 0.1$ & $0.8 \pm 0.2$ & $0.8 \pm 0.2$ \\
\hline WBC $\left(\times 10^{3} / \mathrm{mL}\right)$ & $7.8 \pm 1.8$ & $7.4 \pm 1.8$ & $7.5 \pm 1.7$ \\
\hline Total cholesterol (mg/dL) & $204.1 \pm 30.5$ & $208.7 \pm 28.4$ & $217.3 \pm 40.2$ \\
\hline LDL cholesterol (mg/dL) & $130.1 \pm 28.9$ & $140.7 \pm 32.0$ & $149.2 \pm 37.6^{*}$ \\
\hline HDL cholesterol (mg/dL) & $41.5 \pm 13.5$ & $39.0 \pm 8.7$ & $38.4 \pm 10.3$ \\
\hline Triglyceride (mg/dL) & $150.2 \pm 62.1$ & $160.5 \pm 58.3$ & $153.7 \pm 55.2$ \\
\hline
\end{tabular}

BMI: Body mass index, SBP: Systolic blood pressure, DBP: Diastolic blood pressure, WBC: White blood cell count, LDL: Low density lipoprotein, HDL: High density lipoprotein.

" $p<0.05$ versus group 1 .

${ }^{\#} p<0.05$ versus group 2 .

from those without significant CAD was high (AUC = 0.955 , Figure 3). If we determined a concentration of $147.70 \mathrm{pg} / \mathrm{mL}$ as cutoff value for plasma IMD, we could predict the presence of CAD with significant stenosis with $88.6 \%$ sensitivity and $88.7 \%$ specificity. The negative

Table 2 Plasma intermedin levels according to cardiovascular risk factors

\begin{tabular}{lcc}
\hline Variable & Intermedin & $\boldsymbol{p}$ \\
\hline Gender & $142.3 \pm 16.8$ & 0.002 \\
Male & $135.3 \pm 18.7$ & \\
Female & & \\
Smoking habit & $143.4 \pm 16.8$ & 0.02 \\
Current smoker & $137.3 \pm 18.3$ & \\
Nonsmokers and ex-smokers & & \\
Hypertension & $138.7 \pm 17.7$ & 0.71 \\
Present & $139.6 \pm 19.0$ & \\
Absent & & \\
Diabetes mellitus & $140.7 \pm 16.9$ & \\
Present & $138.2 \pm 18.9$ & \\
Absent & & 0.78 \\
Hyperlipidemia & $138.1 \pm 18.3$ & \\
Present & $139.0 \pm 19.4$ & \\
Absent & &
\end{tabular}

predictive value was $88.7 \%$. The diagnostic value for plasma IMD levels in discriminating patients with normal coronary arteries, who underwent coronary angiography with suspicion of CAD, was also high. A plasma IMD level of $<126.60 \mathrm{pg} / \mathrm{mL}$ could discriminate a patient with normal coronary arteries from patients with angiographically proven CAD with a sensitivity and specifity of $84.7 \%$, and 83.3\% respectively.

Multivariate linear logistic regression analysis was performed to estimate the effects of plasma IMD levels together with other cardiovascular risk factors such as age, male gender, smoking, BMI, waist circumference, hypertension, diabetes, dyslipidemia and LDL-cholesterol levels on the presence of CAD (Table 4). Plasma IMD levels were independently associated with the presence of CAD $(\beta=0.267, \mathrm{BE}=1.305, p<0.001)$.

\section{Discussion and conclusion}

Roh and colleagues identified a new peptide that possesses similar characteristics with adrenomedullin and named it IMD due to its primarily expression in the intermediate lobe of the anterior pituitary glands [16]. This peptide is a member of the CGRP superfamily, which is well known as an endocrine and neurocrine integrator of homeostasis in the cardiovascular system [17]. Subsequent preclinical and human studies reported that IMD is also localized in the left ventricular myocytes, pericardial adipocytes, in vascular 
Table 3 Angiographical characteristics of the study population and plasma IMD levels of the groups

\begin{tabular}{|c|c|c|c|}
\hline & $\begin{array}{l}\text { Patients without CAD } \\
\text { (Group 1) }(\mathrm{n}=48)\end{array}$ & $\begin{array}{l}\text { Patients with }<50 \% \text { coronary stenosis } \\
\text { (Group 2) }(n=111)\end{array}$ & $\begin{array}{c}\text { Patients with } \geq 50 \% \text { coronary stenosis } \\
\text { (Group 3) }(n=79)\end{array}$ \\
\hline Gensini score & 0 & $17.2 \pm 14.3$ & $152.2 \pm 91.7$ \\
\hline \multicolumn{4}{|l|}{ Number of diseased vessels } \\
\hline 0 & 48 & 50 & 0 \\
\hline 1 & - & - & 18 \\
\hline 2 & - & - & 23 \\
\hline 3 & - & - & 23 \\
\hline 4 & - & - & 2 \\
\hline Vessel score & 0 & 0 & $2.2 \pm 1.0$ \\
\hline \multicolumn{4}{|l|}{ Location of stenosis ( $\geq 50 \%$ ) } \\
\hline LMCA & - & - & 9 \\
\hline$\angle A D$ & - & - & 58 \\
\hline LCX & - & - & 51 \\
\hline RCA & - & - & 53 \\
\hline SYNTAX score & - & - & $15.1 \pm 7.8$ \\
\hline Plasma Intermedin (pg/mL) & $117.6 \pm 7.9$ & $134.8 \pm 11.9^{*}$ & $157.7 \pm 9.6^{*}$, , \\
\hline
\end{tabular}

LMCA: Left main coronary artery, LAD: Left coronary artery, LCx: Left circumflex artery, RCA: Right coronary artery.

* $p<0.01$ compared to group 1 .

\#p $<0.01$ compared to group 2 .

Data were presented as mean \pm standard deviation.

smooth muscle cells of coronary arteries, renal arterioles and tubular cells [6].

Increased expression of IMD in animal models of cardiovascular diseases such as myocardial hypertrophy, chronic hypoxia-induced pulmonary hypertension, ischemiareperfusion injury, hypertension and heart failure suggested a key role of IMD in cardiac pathophysiology $[5,6,18,19]$. The robust increase in expression of the peptide in hyper- trophied and ischemic myocardium indicates an important protective role for IMD as an endogenous counterregulatory peptide in the heart. This was underlined by the observation that progression of atherosclerotic plaque in ApoE null mice was terminated with exogenous administration of IMD [9].

On cell level it has been shown that intermedin protects human macrovascular, microvascular, and cardiac

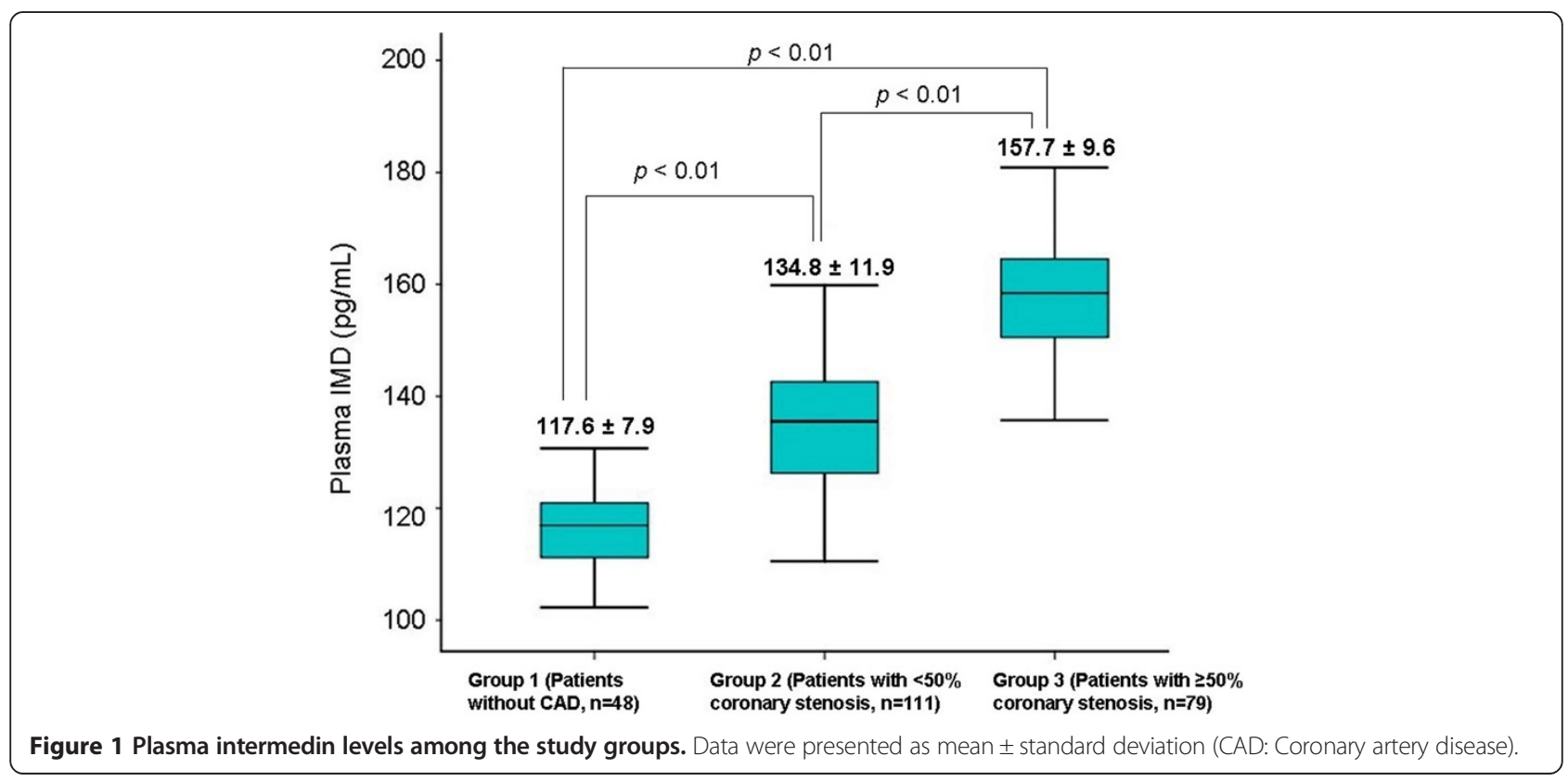




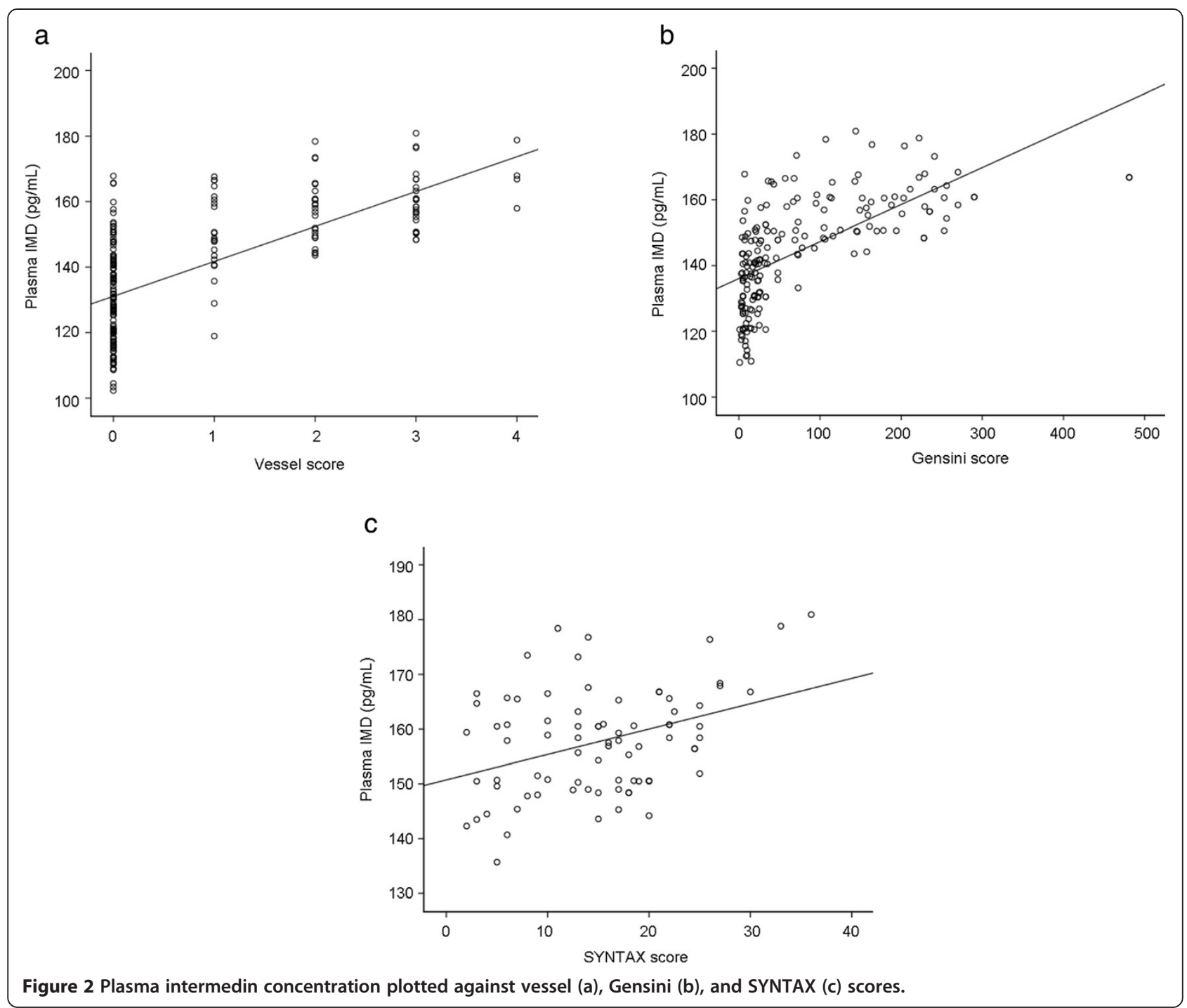

non-vascular cells against ischemia reperfusion injury via AM(1)-receptor signaling [20]. Furthermore, IMD exerts potent cardioprotective effects against acute rat ischemic injury [21], inhibiting endoplasmic reticulum stress via PI3 kinase-Akt signaling [22], and activating cardioprotective Akt/GSK-3beta signaling, decreasing mitochondrialmediated myocardial apoptosis [23].

Considering the cardioprotective effects of endogenous and exogenous IMD, we assumed that plasma IMD levels might be upregulated in stable patients with suspicious $\mathrm{CAD}$, countering the detrimental effects of endothelial dysfunction and oxidative stress related to CAD, even in an early phase of atherosclerotic changes.

So far, plasma levels of IMD in patients with CAD were investigated only in a few studies $[10,11]$. Qin et al. demonstrated that plasma IMD levels were markedly enhanced in forty-one patients, who underwent coronary angiography with suspected acute coronary syndrome (UAP and acute
MI), compared to younger healthy controls [10]. In this patient collective, high age, elevated systolic blood pressure, concentrations of brain natriuretic peptide (BNP), highsensitivity C-reactive protein (hsCRP), creatinine kinase-MB (CK-MB), and Gensini score were correlated to plasma IMD levels. A similar study investigated the serial changes in plasma IMD levels in patients with acute ST-segment elevation MI, stable CAD patients, and healthy controls [11]. Besides the detection of higher values of plasma IMD in patients with acute MI compared to stable patients with CAD, plasma IMD concentration was gradually elevated in patients with CAD compared to healthy to controls (148.8 \pm 6.0 versus $125.9 \pm 9.2 \mathrm{pg} / \mathrm{mL}, p<0.05)$. Significant correlations between IMD and diverse markers of oxidative stress such as superoxide dismutase and malonaldehyde during the course of acute MI supported the hypothesis that IMD might be released to protect the heart from deleterious effects related to ischemia reperfusion injury. The lack of an 


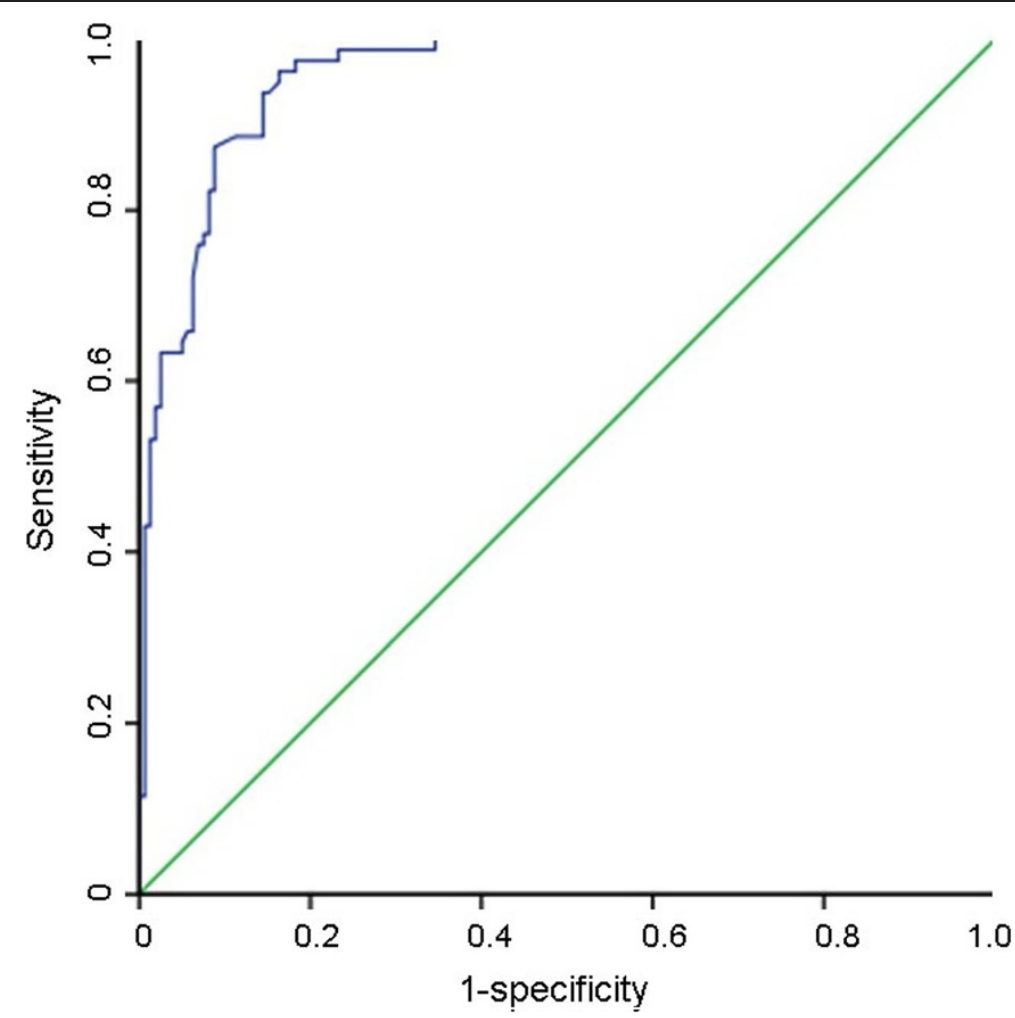

Figure 3 Receiver-operating characteristic (ROC) analysis for plasma intermedin for the discrimination of patients with $\geq 50 \%$ stenosis at least in one of the coronary arteries from patients with normal coronary anatomy and $<50 \%$ stenosis.

association between IMD levels and markers of myocardial injury such as CK-MB, Troponin-T and BNP was explained by the insensitivity of IMD for early diagnosis of MI. Thus IMD seems to be more a biomarker of a chronic process, namely atherosclerosis, helping to determine the presence and extent of CAD in a stable patient collective with suspicious CAD.

Table 4 Multivariate logistic regression analysis of various variables (demographical features, co-morbidities and plasma intermedin levels) which determined as predictors of coronary artery disease in univariate analyses $\left(R^{2}=0.63\right)$

\begin{tabular}{lccc}
\hline Variables & $\boldsymbol{\beta}$ Coefficient & Odds (95\% C.I.) & $\boldsymbol{p}$ \\
\hline Current smoker & -19.60 & 0 & 1 \\
BMI & -0.01 & $0.99(0.57-1.70)$ & 0.96 \\
Hypertension & -0.69 & $0.50(0.03-8.03)$ & 0.62 \\
Age & 0.05 & $1.05(0.89-1.23)$ & 0.56 \\
Dyslipidemia & -1.27 & $0.28(0-20.74)$ & 0.56 \\
Male gender & -1.80 & $0.16(0.01-2.16)$ & 0.17 \\
LDL-cholesterol & 0.02 & $1.02(0.99-1.05)$ & 0.18 \\
Diabetes mellitus & 2.08 & $8.03(0.66-98.0)$ & 0.10 \\
Waist circumference & -0.10 & $0.90(0.81-1.01)$ & 0.07 \\
Intermedin & 0.27 & $1.30(1.11-1.53)$ & $<0.001$ \\
\hline
\end{tabular}

BMI: Body mass index, LDL: low-density lipoprotein.
Thus our aim was to evaluate the predictive strength of IMD in selecting stable patients with CAD from healthy individuals, who were admitted to first time diagnostic angiography. We demonstrated that plasma IMD levels were enhanced in patients with angiographically confirmed CAD compared to patients with normal coronary angiograms. In addition, plasma IMD levels were significantly higher in patients with critical coronary artery stenosis $(\geq 50 \%)$ than in patients with noncritical $(<50 \%)$ coronary lesions. There was a positive and significant correlation between severity and extent of CAD assessed with angiographic indexes and plasma IMD levels. Circulating levels of IMD were independently associated with the presence of CAD. Possible potential implications of this study could be regarded as follows:

- measurement of serum IMD levels could support the clinician about the presence and severity of CAD before coronary angiography.

- unnecessary coronary angiographies could be prevented by measuring serum IMD levels.

- as a biomarker of atherosclerotic progression, patients with elevated serum IMD levels could be treated more intensely, for example high dose statins, renin/angiotensin system blockers and 
antiplatelets. Also, close medical monitoring for the patients with elevated serum IMD levels could be reasonable to early detection and treatment of atherosclerotic involvement.

Elevated levels of IMD in patients with CAD seem to be associated with atherosclerotic development, even in patients with a subclinical disease. Besides, correlations between IMD and indices of CAD extent and severity could be regarded as increased expression of IMD in compensatory fashion to the degree of atherosclerotic involvement. Second, for the first time in the literature, our results revealed that plasma IMD levels were independently associated with the presence of CAD. Although we demonstrated significant correlations between the plasma IMD level and Gensini score similar to previous studies, IMD appeared as an independent variable associated with the presence of $\mathrm{CAD}$ when we evaluated the presence of $\mathrm{CAD}$ with other variables such as age, male gender, smoking, and LDL cholesterol in a multivariate logistic regression analysis. Furthermore, we demonstrated the essential attributes of IMD testing such as sensitivity, specifity, positive and negative predictive values for the first-time. These results suggested that IMD could be used as a biomarker to discriminate patients with CAD and predict those with severe disease who require early and intensive therapeutic intervention.

Although the correlation between serum IMD levels and angiographic indices such as vessel and Gensini score was strong ( $r s=0.710$ and $r s=0.742$, respectively), a diminution was stood out in correlation coefficient for SYNTAX score $(\mathrm{rs}=0.296)$. The SYNTAX score is a relatively new index compared to the others and primary focus on coronary anatomy, which includes defining the coronary dominance and characterizing the presence and features of chronic total occlusions, trifurcations, bifurcations, aorto-ostial lesions, severe tortuosity, long lesions, heavy calcification, thrombus and/or diffuse disease [14]. The SYNTAX score originated from the landmark Synergy between PCI with Taxus and Cardiac Surgery (SYNTAX) trial in 2009 which sought to establish whether coronary artery bypass grafting (CABG) or percutaneous coronary intervention (PCI) was the standard of care for patients with three-vessel or left main coronary artery disease. Although the score is used primarily to risk-stratify these complex patients, the use of the score for appropriate risk stratification in order to determine the optimal revascularization strategy in all CAD types are very popular nowadays. In our study, the mean SYNTAX score was very low $(15.1 \pm 7.8)$ due to a few number of patients with complex disease (for example, 2 patients with LMCA stenosis). We assume that poor correlation between SYNTAX score and serum IMD levels was resulted from relatively low SYNTAX scores and limited number of patients with high (>22) SYNTAX score in our study.
The main limitation of our study was that other markers of atherogenesis such as hsCRP, chemotactic molecules, interleukin-6 and growth differentiation factor-15 were not determined. Other pathologies that could potentially elevate serum IMD levels such as gastrointestinal, pituitary, other organ or body systems did not take into account. Also, patients were not investigated for other potential sites of atherosclerosis, such as carotid artery, thoraco-abdominal aorta, renal arteries, and peripheral vascular bed. Although potential underlying mechanisms proposed to explain the elevated plasma IMD levels in patients with CAD were discussed, the exact mechanism remains unclear. Additionally, this was a cross-sectional study and thus, the clinical impact of the results should be assessed by clinical follow-up studies.

\section{Competing interests}

The authors declare that they have no competing interests.

\section{Authors' contributions}

AHY developed the study idea and design, analyzed the data, interpreted the data analysis and has written the manuscript. AB participated in the design of the study and performed statistical analysis. ZI, SK and ES participated in the coordination of the study and helped to draft the manuscript. EA and MN carried out the immunoassays and participated in the data analysis. EE and PJ performed the angiographic analysis and participated in data analysis. OG helped to draft the manuscript, to integrate and interpret the analyzed data. All authors read and approved the final manuscript.

\section{Acknowledgements}

The authors would like to thank staff at the angiography unit for their kind contribution and support while conducting the study. There is not any financial sponsorship, grant or funding.

Received: 24 July 2014 Accepted: 26 November 2014 Published: 11 December 2014

\section{References}

1. Nürnberger J, Kribben A, Philipp T, Erbel R: Arterial compliance (stiffness) as a marker of subclinical atherosclerosis. Herz 2007, 32(5):379-386.

2. Hansson GK, Robertson AK, Söderberg-Nauclér C: Inflammation and atherosclerosis. Annu Rev Pathol 2006, 1:297-329.

3. Giannotti G, Landmesser U: Endothelial dysfunction as an early sign of atherosclerosis. Herz 2007, 32(7):568-572.

4. Libby P, Ridker PM, Hansson GK: Leducq transatlantic network on atherothrombosis: inflammation in atherosclerosis: from pathophysiology to practice. J Am Coll Cardiol 2009, 54(23):2129-2138.

5. Pan CS, Yang JH, Cai DY, Zhao J, Gerns H, Yang J, Chang JK, Tang CS, Qi YF: Cardiovascular effects of newly discovered peptide intermedin/ adrenomedullin 2. Peptides 2005, 26(9):1640-1646.

6. Bell D, McDermott BJ: Intermedin (adrenomedullin-2): a novel counterregulatory peptide in the cardiovascular and renal systems. $\mathrm{Br} J$ Pharmacol 2008, 153(Suppl 1):S247-S262.

7. Yang $X$, Zhang H, Jia Y, Ni L, Li G, Xue L, Jiang Y: Effects of intermedin1-53 on myocardial fibrosis. Acta Biochim Biophys Sin (Shanghai) 2013, 45(2):141-148.

8. Zhao L, Peng DQ, Zhang J, Song JQ, Teng X, Yu YR, Tang CS, Qi YF: Extracellular signal-regulated kinase $1 / 2$ activation is involved in intermedin 1-53 attenuating myocardial oxidative stress injury induced by ischemia/reperfusion. Peptides 2012, 33(2):329-335.

9. Zhang X, Gu L, Chen X, Wang S, Deng X, Liu K, Lv Z, Yang R, He S, Peng Y, Huang D, Jiang W, Wu K: Intermedin ameliorates atherosclerosis in ApoE null mice by modifying lipid profiles. Peptides 2012, 37(2):189-193.

10. Qin YW, Teng X, He JQ, Du J, Tang CS, Qi YF: Increased plasma levels of intermedin and brain natriuretic peptide associated with severity of coronary stenosis in acute coronary syndrome. Peptides 2013, 42:84-88. 
11. Lv Z, Wu K, Chen $X$, Zhang $X$, Hong B: Plasma intermedin levels in patients with acute myocardial infarction. Peptides 2013, 43:121-125.

12. Gensini GG: A more meaningful scoring system for determining the severity of coronary heart disease. Am J Cardiol 1983, 51(3):606.

13. Sullivan DR, Marwick TH, Freedman SB: A new method of scoring coronary angiograms to reflect extent of coronary atherosclerosis and improve correlation with major risk factors. Am Heart J 1990, 119(6):1262-1267.

14. Sianos G, Morel MA, Kappetein AP, Morice MC, Colombo A, Dawkins K, van den Brand M, Van Dyck N, Russell ME, Mohr FW, Serruys PW: The SYNTAX score: an angiographic tool grading the complexity of coronary artery disease. Eurolntervention 2005, 1(2):219-227.

15. Friedewald WT, Levy RI, Fredrickson DS: Estimation of the concentration of low-density lipoprotein cholesterol in plasma, without use of the preparative ultracentrifuge. Clin Chem 1972, 18(6):499-502.

16. Roh J, Chang CL, Bhalla A, Klein C, Hsu SY: Intermedin is a calcitonin/ calcitonin gene-related peptide family peptide acting through the calcitonin receptor-like receptor/receptor activity-modifying protein receptor complexes. J Biol Chem 2004, 279(8):7264-7274.

17. Hinson JP, Kapas S, Smith DM: Adrenomedullin, a multifunctional regulatory peptide. Endocr Rev 2000, 21(2):138-167.

18. Bell D, Zhao Y, McCoy FP, Devine A, McDermott BJ: Expression of the counter-regulatory peptide intermedin is augmented in the presence of oxidative stress in hypertrophied cardiomyocytes. Cell Physiol Biochem 2008, 21(5-6):409-420.

19. Hirose $T$, Totsune $K$, Mori N, Morimoto R, Hashimoto M, Nakashige $Y$, Metoki H, Asayama K, Kikuya M, Ohkubo T, Hashimoto J, Sasano H, Kohzuki M, Takahashi K, Imai Y: Increased expression of adrenomedullin 2/intermedin in rat hearts with congestive heart failure. Eur J Heart Fail 2008, 10(9):840-849.

20. Bell D, Campbell M, Ferguson M, Sayers L, Donaghy L, O'Regan A, Jewhurs $V$, Harbinson M: AM(1)-receptor-dependent protection by intermedin of human vascular and cardiac non-vascular cells from ischaemiareperfusion injury. J Physiol 2012, 590(Pt 5):1181-1197.

21. Du QX, Yue W, Wang YY: Effect and mechanism of intermedin in acute rat cardiac ischemic injury. Fa yi xue za zhi 2011, 27(3):164-168

22. Teng X, Song J, Zhang G, Cai Y, Yuan F, Du J, Tang C, Qi Y: Inhibition of endoplasmic reticulum stress by intermedin(1-53) protects against myocardial injury through a PI3 kinase-Akt signaling pathway. J Mol Med (Berl) 2011, 89(12):1195-1205.

23. Song JQ, Teng X, Cai Y, Tang CS, Qi YF: Activation of Akt/GSK-3beta signaling pathway is involved in intermedin(1-53) protection against myocardial apoptosis induced by ischemia/reperfusion. Apoptosis 2009, 14(11):1299-1307.

doi:10.1186/1471-2261-14-182

Cite this article as: Yamac et al: Implication of plasma intermedin levels in patients who underwent first-time diagnostic coronary angiography: a single centre, cross-sectional study. BMC Cardiovascular Disorders 2014 14:182.

\section{Submit your next manuscript to BioMed Central and take full advantage of:}

- Convenient online submission

- Thorough peer review

- No space constraints or color figure charges

- Immediate publication on acceptance

- Inclusion in PubMed, CAS, Scopus and Google Scholar

- Research which is freely available for redistribution 